\title{
Moyamoya by magnetic resonance imaging scan
}

\author{
Caroline Edward Ayad, Ahmed Alamin Alnoor, Aymen El-Mesallamy
}

\section{CASE REPORT}

A 15-year-old Sudanese male was referred to the Magnetic Resonance Imaging (MRI) department with severe headache. Brain MRI axial $\mathrm{T}_{1}, \mathrm{~T}_{2}$, diffusion, post contrast series, fluid attenuation inversion recovery (FLAIR) and time-of-flight Magnetic Resonance Angiography (TOF-MRA) techniques were obtained. Images showed tiny punctuate signal void in all pulse sequence in both basal ganglia. Also there are a number of collaterals seen surroundings the mid brain and within the supra sellar cistern.

In post-contrast enhancement images there are obvious enhanced collaterals within the sulci of both cerebral hemispheres, the posterior fosse structures including the brain stem appeared intact. The obtained diffusion weighted images showed no evidence of recent ischemic insult. There was no obvious intra axial mass lesion, signal of blood degradation or extra axial fluid collection.

Magnetic resonance angiography (MRA) described both internal carotid arteries; they showed obvious occlusion of their supraclinoid portions without well

Caroline Edward Ayad ${ }^{1}$, Ahmed Alamin Alnoor ${ }^{2}$, Aymen ElMesallamy ${ }^{3}$

Affiliations: ${ }^{1} \mathrm{PhD}$, Diagnostic Radiologic Technology, Sudan University of Science and Technology, Deputy Dean, Radiology Department, College of Medical Radiological Science, Khartoum, Sudan; ${ }^{2}$ B.Sc, Radiological Science, University of Medical Science and Technology, Radiographic Technologist, Radiological Science Department, Faculty of Radiological Science, Khartoum, Sudan; ${ }^{3} \mathrm{MD}$, Consultant Radiologist, Radiology Department, Alzaytouna Specialist Hospital, Khartoum, Sudan.

Corresponding Author: Caroline Edward Ayad, Sudan University of Science and Technology, College of Medical Radiological Science, Khartoum, Sudan P.O. Box 1908, Khartoum, Sudan; Ph: +249 0922044764; Email: carolineayad@yahoo.com

Received: 18 March 2013

Accepted: 23 April 2013

Published: 01 April 2014 formed circle of Willis, besides tortuous collaterals seen surrounding cistern forming A net-like appearance, the vertebral arteries as well as the basilar artery were intact.

An MRA revealed bilateral supraclinoid internal carotid arteries occlusion without well formed circle of Willis and collateralization as moyamoya disease (Figure 1).
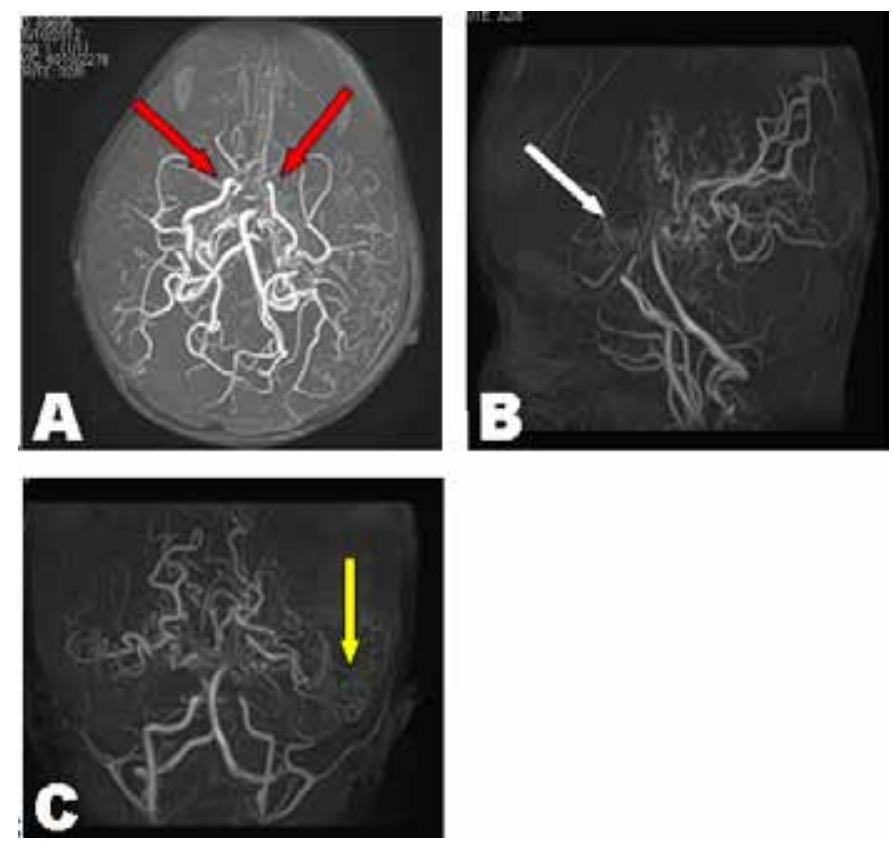

Figure 1: Magnetic resonance angiography axial, sagittal, coronal brain images of a 15-year-old Sudanese child showing Moyamoya Disease (A) Both internal carotid arteries explain obvious occlusion (red arrows) of their supra clinoid portion, (B) Circle of Willis (white arrow) is not well formed, (C) Tortuous collaterals are seen surrounding cistern forming net like appearance (yellow arrow).

\section{DISCUSSION}

Moyamoya is a rare cerebrovascular disease among Japanese [1]. Ethnicity-incidence ratios for Whites as compared to Asian-Americans were 1:4.6, and as compared to African-Americans was 1:2.2. To the best 
of our knowledge, no similar cases have been reported in Sudanese population in existing literature [2]. The pathogenesis of moyamoya is idiopathic progressive arteriopathy of childhood; where occlusion of the circle of Willis, narrowing of distal internal carotid artery (ICA) and proximal circle of Willis vessels with secondary collateralization are detected the disease is poorly understood and may be due to genetic and environmental factors [3, 4]. The diagnosis of moyamoya disease can be diagnosed by different imaging modalities and radiographic evaluation and it primarily depends on angiographic results, including occlusion of the supraclinoid ICA and formation of extensive collateral vessels $[1,3]$. Several previous studies have revealed that Moyamoya disease can be identified on contrastenhanced computed tomography (CT) or MRI scans, owing to their sensitivity to ischemic changes [5-7].

Three-dimensional CT angiography have limitations in diagnosis of moyamoya disease because of the limited spatial resolution, difficulty in covering the whole intracranial vasculature network of leptomeningeal anastomotic channels [1]. The assessment of cerebral ischemia associated with moyamoya by diffusionweighted MRI scan has value in disease evolution [8]. Infarctions are better delineated with $\mathrm{T}_{1}-$ and $\mathrm{T}_{2}$ - imaging $[9,10]$. The MRI findings in $\mathrm{T}_{1}$ are multiple dot-like flow voids in basal ganglia, $\mathrm{T}_{2}$-weighted images are of high signal small vessel cortical and white matter infarcts, collateral vessels appear as "net-like" cisternal filling defects , FLAIR shows Bright sulci at leptomeningeal "ivy sign"[3]. Another MR protocols are useful in moyamoya; $\mathrm{T}_{2}$ star gradient recall echo is useful prior hemorrhage , $\mathrm{T}_{1}$ with contrast shows lenticulostriate collaterals with enhancing "dots" in basal gangila and "net-like" thin vessels in cisterns, Leptomeningeal enhancement gives "ivy sign", MR spectroscope shows Lactate in acutely infarcted tissues and $\mathrm{NAA} / \mathrm{Cr}$ and $\mathrm{Cho} / \mathrm{Cr}$ ratios frontal white matter increase after revascularization, perfusionweighted imaging (PWI) shows low perfusion deep hemispheric white matter, is relatively high perfusion posterior circulation, PWI may be abnormal even if MRI scan is normal [3].

Moyamoya on MRI is Characterized by diminishing of flow voids in the internal carotid and middle and anterior cerebral arteries together with collateral flow voids in the basal ganglia and thalamus [11-13].

Magnetic resonance angiography has been used to demonstrate the intracranial vessels, where it is non invasive, no contrast media, and ionizing radiation is used. MRA is useful in the diagnosis of moyamoya disease as mentioned by Yamada et al. [14]. MRI scan has been proposed to be used instead of conventional cerebral angiography [12].

\section{CONCLUSION}

Regardless of the excellent diagnostic value, broad imaging protocols and noninvasive nature, it has been proposed that magnetic resonance angiography should be used as a diagnostic imaging modality for moyamoya disease.

\section{How to cite this article}

Ayad CE, Alnoor AA, El-Mesallamy A. Moyamoya by magnetic resonance imaging scan. International Journal of Case Reports and Images 2014;5(4):320322.

doi: 10.5348/ijcri-201454-CL-10042

$* * * * * * * * *$

\section{Author Contributions}

Caroline Edward Ayad - Substantial contributions to conception and design, Acquisition of data, Analysis and interpretation of data, Drafting the article, Revising it critically for important intellectual content, Final approval of the version to be published

Ahmed Alamin Alnoor - Analysis and interpretation of data, Revising it critically for important intellectual content, Final approval of the version to be published Aymen El-Mesallamy - Analysis and interpretation of data, Revising it critically for important intellectual content, Final approval of the version to be published

\section{Guarantor}

The corresponding author is the guarantor of submission.

\section{Conflict of Interest}

Authors declare no conflict of interest.

\section{Copyright}

(C) Caroline Edward Ayad et al. 2014; This article is distributed under the terms of Creative Commons attribution 3.0 License which permits unrestricted use, distribution and reproduction in any means provided the original authors and original publisher are properly credited. (Please see www.ijcasereportsandimages.com/ copyright-policy.php for more information.)

\section{REFERENCES}

1. Tsuchiya K, Makita K, Furui S. Moyamoya disease: Diagnosis with three-dimensional CT angiography. Neuroradiology 1994 Aug;36(6):432-4.

2. Uchino K, Johnston SC, Becker KJ, Tirschwell DL. Moyamoya disease in Washington State and California. Neurology 2005 Sep 27;65(6):956-8.

3. Osborn Anne G, Hedlund Gary L, Blaser Susan I, (eds), 2004.Diagnostic imaging Brain. First edition. Canada. Salt Lake City, Utah, AMIRSYS (LTD), ,I,4,46,pp283-6. 
4. Smith Edward R, Scott Michael R. Moyamoya: Epidemiology, presentation, and diagnosis. Neurosurg Clin N Am 2010 Jul;21(3):543-1.

5. Takahashi M, Miyauchi T, Kowada M. Computed tomography of moyamoya disease: Demonstration of occluded arteries and collateral vessels as important diagnostic signs. Radiology 1980 Mar;134(3):671-6.

6. Asari S, Satoh T, Sakurai M, Yamamoto Y, Sadamoto $\mathrm{K}$. The advantage of coronal scanning in cerebral computed angio tomography for diagnosis of moyamoya disease. Radiology 1982 Dec;145(3):70911.

7. Fujisawa I, Asato R, Nishimura K, et al. Moyamoya disease: MR imaging. Radiology 1987 Jul;164(1):1035 .

8. Yamada I, Himeno Y, Nagaoka T, et al. Moyamoya disease: Evaluation with diffusion-weighted and perfusion echo-planar MR imaging. Radiology 1999 Aug;212(2):340-7.

9. Chabbert V, Ranjeva JP, Sevely A, Boetto S, Berry I, Manelfe C. Diffusion- and magnetisation transfer-weighted MRI in childhood moya-moya. Neuroradiology 1998 Apr;40(4):267-71.
10. Fujiwara H, Momoshima S, Kuribayashi S. Leptomeningeal high signal intensity (ivy sign) on fluid-attenuated inversion-recovery (FLAIR) MR images in moyamoya disease. Eur J Radiol 2005 Aug;55(2):224-30.

11. Fujita K, Shirakuni T, Kojima N, Tamaki N, Matsumoto S. Magnetic resonance imaging in moyamoya disease. No Shinkei Geka 1986 Mar;14(3 Suppl):324-30. [Article in Japanese].

12. Yamada I, MatsushimaY, Suzuki S. Moyamoya disease: Diagnosis with three-dimensional time-offlight MR angiography. Radiology 1992 Sep;184(3):773-8.

13. Sunaga Y, Fujinaga T, Ohtsuka T. MRI findings of moyamoya disease in children. No To Hattatsu 1992 Jul;24(4):375-9. [Article in Japanese].

14. Yamada I, MatsushimaY, Suzuki S. Moyamoya disease: Diagnosis with three-dimensional time-of-flight MR angiography. Radiology 1992 Sep;184(3):773-8.
Access full text article on other devices

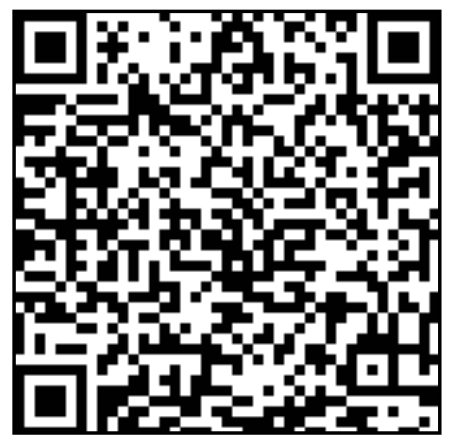

Access PDF of article on other devices

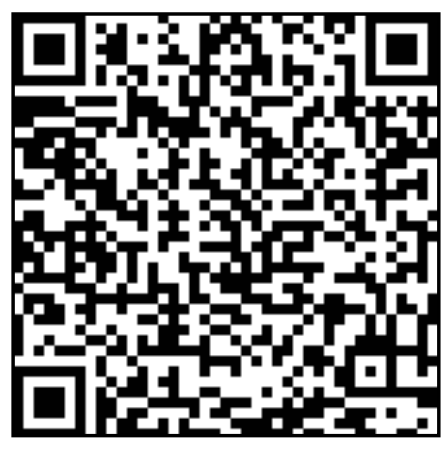

